\title{
PERIODIC VARIATIONS IN THE IUE DATA OF HD 50896
}

\author{
C. LLOYD and D.J. STICKLAND \\ Rutherford Appleton Laboratory, Chilton, U.K.
}

\begin{abstract}
A periodogram analysis of the radial velocities and absorption line strengths from IUE spectra of HD 50896 show evidence of the 3.766 day period usually associated with this star. The dominant line variations occur on time scales of $\sim 1$ day but may be interpreted as higher order harmonics of the 3.766 day period.
\end{abstract}

Key words: stars: Wolf-Rayet - variable - individual: HD 50896

HD 50896 is well known for its 3.766 day variations in light, line fluxes and radial velocities. Although this period is seen in most data sets the variations are complicated and change with time (see Antokhin et al. 1994 for a recent summary). The star has been considered as the most likely candidate for a $\mathrm{WR}+\mathrm{c}$ system, but this interpretation is dogged by the low $\mathrm{X}$-ray emission and the lack of any observable, periodic effect of X-rays on the line profiles, and so rotation or intrinsic wind variations appear a more likely cause.

Two sets of observations have been studied. The first consists of 130 short-wavelength IUE spectra obtained almost continuously over 6 days in December 1988. The data are described in detail and extensively studied by St-Louis et al. (1993). Radial velocities were measured by St-Louis et al. using cross correlation for a region from $1260-1480 \AA$ and centred on the N IV $1718 \mathrm{P}$ Cygni line. A second, rather sparser set of radial velocities, measured in the same manner, comes from a series of IUE SWP spectra obtained over 7 days in 1983 (Willis et al. 1989). The N IV absorption equivalent widths have also been studied. These mimic the $\mathrm{C}$ IV and He II variations but appear less noisy and rather sharper, so are probably the most difficult to model. The absorption variations are also much more rapid than the emission line variations, which seem to more closely resemble the velocity variations. The time variability has been investigated using a least squares sine periodogram which gives the reduction in $\chi^{2}$ for a series of trial frequencies.

The periodograms of the radial velocity measurements are dominated by a frequency $f \sim 0.27$ cycle $\mathrm{d}^{-1}$ which is consistent with the well known period of 3.766 days. In the $1988,1260-1480 \AA$ radial velocities, two less

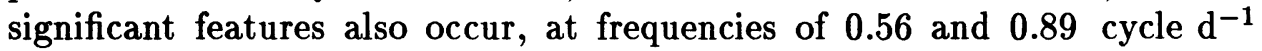
which are very nearly $2 f$ and $3 f$. In fact a Fourier series with 3 terms fits the data just as well. There is nothing at $4 f$. The $1988 \mathrm{~N}$ IV velocities are

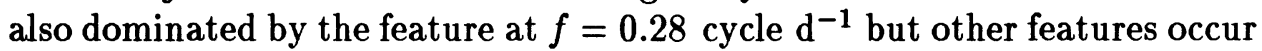

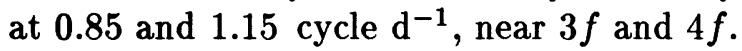

The periodogram of the 1983,1260 - $1480 \AA$ radial velocities, show frequencies of 0.26 and 0.56 cycle $^{-1}$ and there is a weak third frequency at 0.9 cycle $\mathrm{d}^{-1}$. Again the data are equally well fitted by $f, 2 f$ and $3 f$. The 1983 


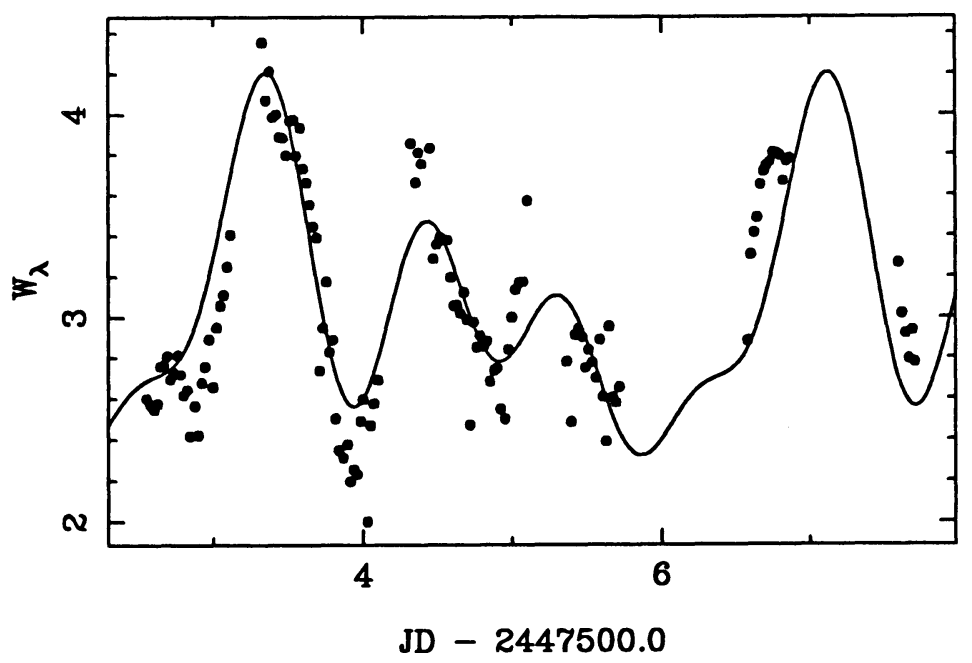

Fig. 1. The N IV absorption equivalent widths from St-Louis et al. fitted with the 3.766 day period plus 3 harmonics. The fit is not perfect but remarkable given that the period was imposed.

$\mathrm{N}$ IV velocities are considerably noiser but the main frequencies are again

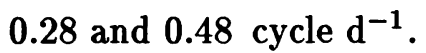

The radial velocities may be well represented by a fundamental frequency corresponding to the well known period of 3.766 days plus its harmonics. There are significant variations on short time scales, corresponding to the higher harmonics, (see St-Louis et al. Fig. 9) so it is difficult to interpret these as true velocity variations. The optical light variations are also now interpreted in terms of harmonics (Antokhin et al. 1994).

The periodogram of the NIV absorption equivalent widths contains 4 features above the noise. In order of decreasing significance these occur at $f \sim 0.87,1.25,0.62$ and 0.31 cycle $\mathrm{d}^{-1}$, and bear a striking resemblance to those of the radial velocity variations. The dominant equivalent width variations occur on a time scale of $\sim 1$ day, which is characteristic of the flow time, and while this has been seen before, the possibility that these are harmonics of, or modulated by, the 3.766 day period has not previously been noticed.

It is a pleasure to thank Nicole St-Louis for access to her measurements.

\section{References}

Antokhin I., Bertrand J-F., Lamontagne R., Moffat A.F.J. 1994, AJ 107, 2179

St-Louis N.S., Howarth I.D., Willis A.J., Stickland D.J, Smith L.J., Conti P.S., Garmany C.D. 1993, $A \& A$ 267, 449

Willis A.J., Howarth I.D., Smith L.J., Conti P.S., Garmany C.D. 1989, A\&A Suppl. 77, 269 